\title{
SISTEMA GERENCIADOR DE BANCO DE DADOS: UM ENSAIO A PARTIR DE DADOS AMBIENTAIS DA BAÍA SUL, FLORIANÓPOLIS(SC).
}

\author{
RODRIGUES, R.M.; PAES, J.V.; DIEHL, F.L.; PINTO, D.F.; ARAÚJO, S.A. de; ABREU, \\ J.G.N. \& L.C. INOCENCIO \\ Universidade do Vale do Itajaí - UNIVALI \\ Centro de Ciências Tecnológicas da Terra e do Mar - CTTMar \\ Rua Uruguai, 458, Cx. P. 360 Itajaí/SC. Fone /Fax (047)3147578 \\ E-mail: rosanasr@mbox1.univali.rct-sc.br
}

\begin{abstract}
RESUMO
O presente trabalho descreve a estruturação de um Banco de Dados Relacional (BDR) a partir dos dados ambientais coletados e analisados pelo Centro de Ciências Tecnológicas da Terra e do Mar - CTTMar - UNIVALI, no projeto "Monitoramento Ambiental da Área de Abrangência das Obras da Via Expressa SC - Sul, Florianópolis/SC". Utilizou-se o aplicativo ACCESS (Microsoft versão $7.0,1994)$ para a definição da arquitetura, estrutura e funcionamento dos relacionamentos entre os dados. Foram realizadas consultas, criados formulários e introduzida a estrutura gráfica no BD, o qual demonstrou estar atendendo às expectativas quanto a sua funcionabilidade.
\end{abstract}

Palavras-chave: Banco de Dados; Dados Ambientais; ACCESS; SIG

\section{MANAGEMENT SYSTEM DATA BASE: A TEST THROUGH ENVIRONMENTAL DATA OF THE SOUTHERN BAY - FLORIANÓPOLIS, SANTA CATARINA STATE}

\begin{abstract}
This paper describes the elaboration of a DATA BASE. During the generation of this bank, environmental parameters (physical, chemical, geological and biological data) have been applied. These data were collected and analyzed by the Center of Technological Sciences of the Earth and Sea. The data are part of the project: "Environmental Monitoring of the Area of Inclusion of the Works of the Highway SC, Florianópolis/SC". The architecture, structure and functioning of the relation among parameters were defined by using the software ACCESS (Microsoft 7.0, 1994). Preliminary consults were done in order to validate the functionality of the Data Base. After that, the graphical structure was introduced and the formularies were created.
\end{abstract}

Key words: Data Base; Environmental Data; ACCESS; GIS.

\section{INTRODUÇÃO}

A reunião de grande número de dados provenientes de fontes e naturezas diversas, o seu relacionamento e análise, sempre foram tarefas árduas. Com a evolução da informática e a sua integração com as diver- sas ciências, foi possível otimizar a manipulação simultânea de tais dados através dos Sistemas Gerenciadores de Bancos de Dados (SGBD).

Os Bancos de Dados (BD), de uma forma geral, têm a função de armazenar, recuperar e analisar informações, ou seja, tem a 
capacidade de gerenciar dados de fontes diversas utilizando-se de tabelas, consultas, formulários e relatórios, sendo "ferramentas" desenvolvidas para auxiliar na manipulação e recuperação de dados de forma eficiente. Desta forma, "o sistema gerenciador de banco de dados é composto de uma coleção de arquivos inter-relacionados e de um conjunto de programas que permitem ao usuário fazer o acesso e a modificar esses arquivos" (KORTH \& SILBERSCHATZ, 1995).

O presente estudo constitui-se num ensaio que se utiliza desta "ferramenta" para organizar, analisar e estruturar de forma relacional dados ambientais gerados pelo projeto "Monitoramento Ambiental da Área de Abrangência das Obras da Via Expressa SCSul, Florianópolis/SC". A estrutura desenvolvida na operacionalidade do BD permite sua atualização e implementação periódica com novas informações. O aplicativo ACCESS (Microsoft versão 7.0, 1994) foi escolhido para o desenvolvimento do trabalho por possuir uma interface amigável e interativa, não sendo necessário qualquer tipo de programação e apresentando recursos para definição e consistência dos dados. A estrutura do trabalho consistiu em criar o banco de dados relacional propriamente dito, ou seja, sua arquitetura, estrutura e funcionamento dos relacionamentos; gerar formulários e introduzir a estrutura gráfica. Como resultado, foi obtido sucesso nas consultas realizadas, demostrando que a arquitetura lógica é funcional para o objetivo proposto. Ressalta-se que este trabalho refere-se à primeira etapa de um projeto maior, no qual os resultados questionados ao $\mathrm{BD}$ serão o elo de ligação entre o SGBD e o SIG (Sistema de Informações Geográficas). Os SIGs são considerados "ferramentas" que têm por função manipular e analisar dados de fontes e formatos diferentes em um determinado espaço geográfico referenciado por coordenadas geográficas. A etapa de elaboração de mapas temáticos de batimetria, geomorfologia, sedimentologia, hipsometria, declividade, uso e ocupação do solo que envolve a Baia Sul está sendo desenvolvida em escala pertinente a um estudo detalhado, utilizando-se o SIG. Cartas analíticas serão geradas a partir dos dados resultantes do BDR com mapeamentos temáticos da área, obtendo-se assim, por meio dos algoritmos lógicos implementados no SIG, a simulação do espaço real. Segundo FERREIRA (1993), "Estabelecer quais aspectos do mundo real interessam e são de possível modelagem não é uma operação simples, principalmente quando este mundo real que se pretende modelar é o complexo meio ambiente". Portanto, a fase em que o trabalho se encontra é a de maior desafio e a meta é elaborar mapas síntese que reflitam a realidade a partir das informações armazenadas no SGBD.

\section{METODOLOGIA}

O presente estudo foi estruturado metodologicamente obedecendo 5 etapas, sendo que quatro destas compõem o presente trabalho, enquanto que a última está em fase de realização, conforme mostra a figura 1.

\section{1- MODELAGEM DOS DADOS AMBIENTAIS}

Em seu trabalho sobre modelagem de dados geográficos, DAVIS (1998) afirma que " O modelo de dados procura sistematizar 0 entendimento a respeito de objetos e fenômenos do mundo real, visando representálos em um sistema informatizado... Assim, é necessário desenvolver uma abstração de objetos e fenômenos do mundo real concebendo uma representação simplificada, mas adequada às finalidades das aplicações que o banco de dados terá."

Para a construção da modelagem neste trabalho, ou seja, na sistematização dos dados dentro da estrutura lógica concebida para o BD, partiu-se das informações ambientais contidas nos relatórios dos anos 




Figura 1 - Diagrama das etapas de desenvolvimento do trabalho.

de 1996 e 1997 elaborados pela equipe do CTTMar. Esta técnica é conhecida como engenharia reversa ou reengenharia, pois objetiva obter um modelo a partir de um produto existente.

Os parâmetros ambientais que compõem o BDR são os seguintes:

1. Parâmetros físico-químicos - profundidade $(\mathrm{m})$; transparência $(\mathrm{m})$; material em suspensão (mg/l); salinidade (psu/78); temperatura $\left({ }^{\circ} \mathrm{C}\right)$;oxigênio dissolvido $(\mathrm{mg} / \mathrm{l}) ; \mathrm{pH}$; nitrito $(\mathrm{mM})$; nitrato $(\mathrm{mM})$; amônia $(\mathrm{mM})$; NID (mM); fósforo (mM); PO fósforo orgânico (mM); PT (mM); clorofila-a (mg/l); DBO-5 (mg/l); óleos (mg/l).

2. Acompanhamento das modificações texturais do fundo - classificação textural; teores de carbonato; teores de matéria orgânica.

3. Turbidez e dispersão de sedimentos finos em suspensão - turbidez na superfície (mg.l ${ }^{1}$ ); turbidez próxima ao fundo (mg. l-1 $^{-1}$; turbidez média da coluna de água (mg.t-1); coeficiente de estratificação; diferencial vertical de

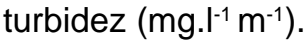

4. Comunidades bentônicas - Anomalocardia brasiliana (indivíduos $/ \mathrm{m}^{2}$ ); biomassa $/ \mathrm{m}^{2}$; comprimento; peso.

5. Comunidades planctônicas - análise do zooplâncton (\% dos grandes grupos do zooplancton); total ( $n^{\circ}$ organismo $\left./ \mathrm{m}^{3}\right)$.

6. Comunidades fitoplanctônicas - densidade de células de organismos fito e zooplanctônicos.

7. Análise microbiológica - NMP/100ml de coliformes fecais.

8. Monitoramento de ictiofauna - relação das espécies.

9. Monitoramento da carcinofauna - freqüên- 
cia absoluta; amplitude e comprimento dos crustáceos decápodas.

10. Monitoramento do manguezal - espécies vegetais presentes.

\section{1 - Decomposição de tabelas}

Segundo BITTENCOURT (1997), "O processo de construção de um modelo é um processo incremental, isto é, um modelo de sistema não é construído em um único passo mas em muitos passos pequenos, pequenas transformações do modelo inicial até o modelo completo". Utilizou-se as tabelas geradas pelos relatórios do projeto que foram decompostas em novas tabelas normalizadas até se chegar ao total que compõem o banco de dados e, consequentemente, aos relacionamentos entre elas.

As tabelas normalizadas e que encontram-se no BD são as seguintes: Projeto; Equipe Técnica; Integrantes; Atividades; Parâmetros Analisados; Estações Amostrais; Coletas;Itens Coletados; Resultados Finais.

\section{2- Definição dos atributos das tabelas}

Após o levantamento dos dados contidos nas tabelas originais foram criadas as tabelas normalizadas, objetivando-se a composição das tabelas propriamente ditas, ou seja, a inserção dos atributos. da:

Composição de cada tabela normaliza-

PROJETO: Código do Projeto; Nome do Projeto; Data de Início do Projeto.
EQUIPE TÉCNICA: Código da Equipe Técnica; Código do Projeto; Nome da Equipe Técnica.

INTEGRANTES DA EQUIPE TÉCNICA: Código do Integrante, Código da Equipe Técnica; Nome do Integrante; Titulação do Integrante; e-mail do Integrante.

ATIVIDADES: Código da Atividade; Código do Projeto; Nome da Atividade.

PARÂMETROS ANÁLISADOS: Código do Parâmetro; Código da Atividade; Descrição do Parâmetro.

ESTAÇÕES AMOSTRAIS: Código da Estação; Código do Projeto; Descrição da Estação; Latitude da Estação; Longitude da Estação.

COLETAS: Código da Coleta; Código do Projeto; Data da Coleta.

ITENS COLETADOS: Código do Item; Código da Coleta; Código do Parâmetro; Código da Estação.

RESULTADOS: Código do Resultado; Código do Item; Resultado Numérico; Resultado Alfa/Numérico.

\section{3 - Relacionamentos entre as tabelas normalizadas}

Definidos os atributos de todas as tabelas, buscou-se relacioná-las afim de possibilitar as consultas e gerar os resultados esperados, conforme dispostos na figura 2.

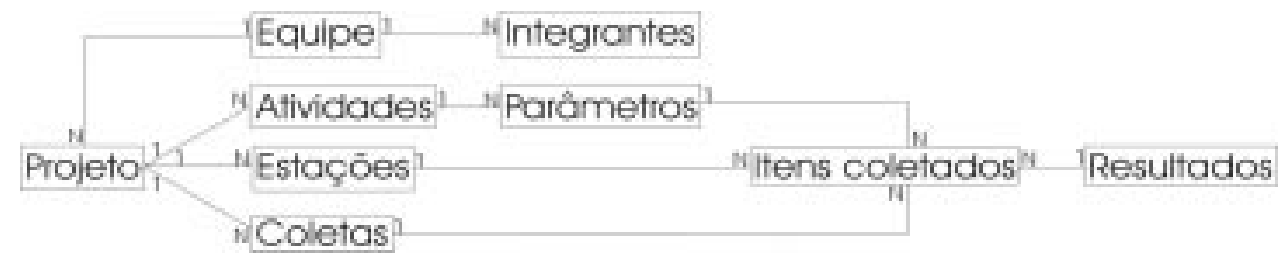

Figura 2 - Diagrama de relacionamentos (estrutura funcional do BDR). 


\section{2- INSERÇÃO DOS DADOS AMBIENTAIS NAS TABELAS}

Normalizadas as tabelas e estabelecidos os prováveis relacionamentos, foram introduzidas as informações ambientais. Após a inserção, realizou-se algumas consultas preliminares para verificar a funcionalidade do Banco de Dados.

\section{3- CONSULTAS}

Nesta etapa de consultas ao BD, todas as informações foram reunidas e ordenadas em diversas tabelas para ordená-las em campos, agrupando informações, de modo a obter cálculos matemáticos, estatísticas e determinar critérios complexos para pesquisas etc.

Por meio das consultas é que se encontram as informações em um BD. Rodar consultas é simplesmente fazer perguntas ao BD. Quando esta é realizada, são especificados os campos desejados, o que se está procurando e quais os campos que se deseja obter. As consultas são efetuadas diretamente ao $\mathrm{BD}$ através de perguntas objetivas sobre qualquer informação contida no SGBD ou ainda, perguntas relacionando os dados.

\section{4- FORMULÁRIOS E GRÁFICOS}

No processo de construção do Banco de Dados foram elaborados formulários, que servirão para visualizar os registros de uma forma organizada e lógica, incluindo gráficos, mapas etc. Estes formulários são uma interface gráfica para auxiliar na entrada de informações e na visualização das consultas e tabelas.

\section{5- INTEGRAÇÃO DOS DADOS ENTRE O SGBD E O SIG}

Esta fase encontra-se em desenvolvimento, utilizando-se o Sistema de Informações Geográficas SPRING (Sistema de
Processamento de Informações Georeferenciadas) para a elaboração dos dados temáticos, que serão posteriormente integrados às informações do SGBD.

\section{RESULTADOS}

Abaixo encontram-se demonstrados alguns exemplos de operacionalidade do BDR:

Exemplo 1 - "Qual o número total de organismos zooplanctônicos $/ \mathrm{m}^{3}$ encontrados nas estações amostrais nos meses de outubro de 1996, janeiro e março de 1997 ? ". A resposta foi apresentada sob a forma de formulário e gráfico (Fig. 3):

A figura 3 (modo formulário) traz uma caixa de listagem com os resultados do total de organismos $/ \mathrm{m}^{3}$ coletados nas estações, com suas respectivas latitudes e longitudes, nas datas especificadas na consulta, além de um gráfico comparativo dos resultados de cada estação.

Outra forma de representação do mesmo resultado é o modo folha de dados ou tabela (Fig. 4).

Exemplo 2: "Qual a classificação textural dos pontos amostrais que sofreram coletas em janeiro de 1997 ?". A resposta foi apresentada sob a forma de um formulário (Fig. 5).

Exemplo 3: "Qual o índice de salinidade e estações amostrais em que a concentração de Amônia (NH4) é menor que $20 \mathrm{mM}$, nas coletas de 1996 e 1997 ?". A resposta é apreentada sob forma de tabela (Fig. 6).

\section{CONSIDERAÇÕES FINAIS}

$O$ presente estudo constitui-se num ensaio, estando o BDR apto para a implementação com novos dados. Salienta- 
se que um Banco de Dados deve ser constantemente utilizado, disponibilizado e sempre implementado com novas informações.

A concepção do trabalho foi o de integrar o BDR com o Sistema de Informações

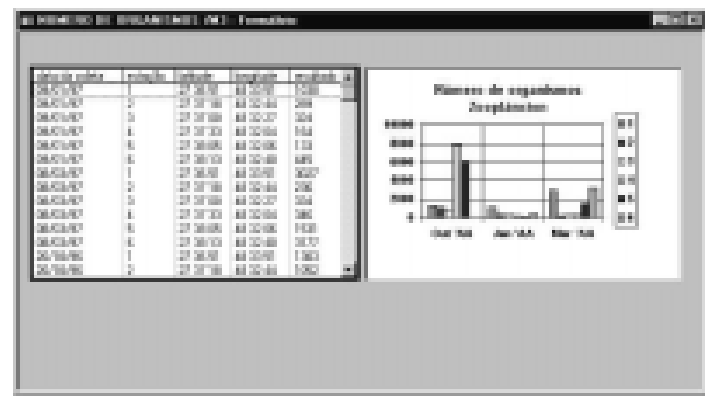

Figura 3 - Formulário com a tabela e gráfico demonstrativo do número de organismos zooplanctônicos $/ \mathrm{m}^{3}$.



Figura 4 - Resultado da consulta do número total de organismos zooplanctônicos $/ \mathrm{m}^{3}$ nas estações amostrais em modo folha de dados ou tabela.

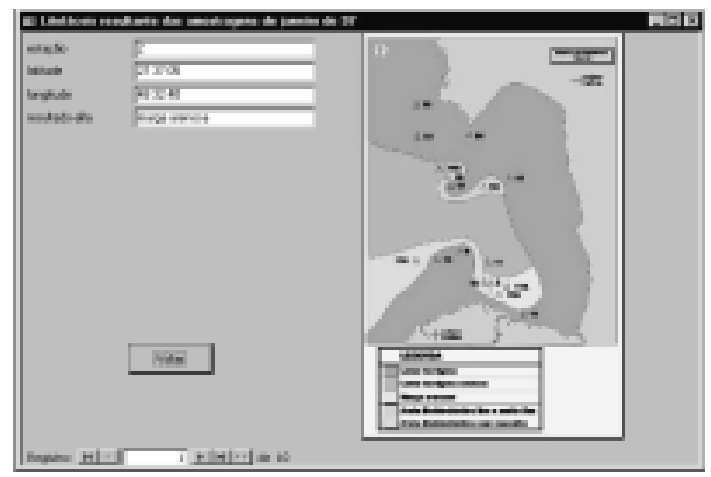

Figura 5 - Formulário contendo mapa de litofáceis de fundo gerado pela coleta amostral de janeiro de 1997.

\begin{tabular}{|c|c|c|c|c|c|}
\hline \multicolumn{6}{|c|}{ 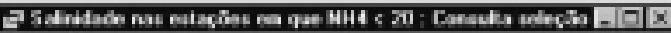 } \\
\hline & data da colata & esalaçio & Teatude & longilude & resulfato \\
\hline \multirow[t]{5}{*}{ 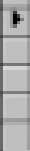 } & इ्गाक & s & 27351 & 483351 & 343 \\
\hline & 080397 & $\mathbf{x}$ & 27351 & 489351 & $\mathbf{2 0 3}$ \\
\hline & 2010\% & 5 & 27351 & 4A 3551 & 32.5 \\
\hline & 000100 & & $\mathbb{2} \mathbf{3} 13$ & 403240 & 327 \\
\hline & Denos:97 & & 273813 & 483240 & 29.5 \\
\hline & $\ln 14 \mid \mathrm{I}$ & $3+$ & Wu be s & & \\
\hline
\end{tabular}

Figura 6 - Tabela contendo o índice de salinidade das estações em que a concentração de NH4 é menor que $20 \mathrm{mM}$.

Geográficas (SIG) a partir dos dados resultantes das consultas com informações ambientais geo-referenciados. Dados georeferenciados, quantificados, analisados e com formatos de saída gráfica (inclusive sob a forma de mapas temáticos), somente podem ser fornecidos por SIGs. Ressalta-se que a estruturação e relacionamento das informações têm fornecido respostas satisfatórias, o que irá possibilitar a geração de mapas síntese que simulam a realidade ambiental da área de estudo.

\section{REFERÊNCIAS BIBLIOGRÁFICAS}

Bittencourt, G.R. 1997 Banco de Dados II (apostila de curso) Ciências da Computação UNIVALI, 50p. Itajaí, SC.

Davis. C. 1998. Modelagem de Dados Geográficos. InfoGeo, 2:38-40.

Ferreira, C.R. 1993. Projeto Lógico de uma Base de Dados para Aplicação em Sistema de Informação Geo-Ambiental. Anais XVI Congresso Brasileiro de Cartografia, p.747-756,

Korth, H. F. \& Silberschatz, A. 1995. Sistema de Banco de Dados. Ed. Makron Books do Brasil, 754p.

Machado, N.R. \& Abreu,M. 1996. Projeto Banco de Dados - Uma Visão Prática. $3^{\mathrm{a}}$ ed. Editora Érica 298 p.

Manual - Microsoft Corporation Microsoft Access 1994. Sistema de Gerenciamento de Banco de Dados Relacional para Windows Versão 2.0. 458p. 
Palmier, S. 1994. Access 2 for Windows. Ed. Berkeley Brasil, 290p.

UNIVALI/CTTMar. 1997. Relatório Técnico do Projeto Monitoramento Ambiental da
Área de Abrangência das Obras da Via Expressa SC - Sul, Florianópolis/SC. 80p. 